\title{
Research of Automatic Disturbance Rejection Controller for Fiber Optic Gyro Servo Stabilized System
}

\author{
Qing Zhang, Li-dong Guo, Liang Huo \\ College of Automation, Harbin Engineering University, HEU, Harbin, China \\ zhq402@hrbeu.edu.cn, guolidong@hrbeu.edu.cn, huoliang@hrbeu.edu.cn
}

\begin{abstract}
In order to overcome the deficiencies of classic control method in the disturbance rejection and robustness, the automatic disturbance rejection controller (ADRC) was introduced into the fiber optic gyro(FOG) stabilized platform. A control model for the position servo loop of FOG stabilized platform was set up. The non-linear structure of ADRC was discussed. ADRC was designed to improve the dynamic performance indexes of FOG stabilized platform. Considering the changing parameters and disturbing factors that may appear in real work, the quality of disturbance rejection and robustness of the ADRC was tested. The ADRC and PID controller were simulated in the same conditions. Simulations show that the oscillations and overshoots of ADRC was not obvious. ADRC has faster and more precise responses. ADRC is not sensitive to the changes of the model parameters.
\end{abstract}

Keywords - proportional-integral-derivative (PID); automatic disturbance rejection controller (ADRC); servo system; controller simulation (key words)

\section{INTRODUCTION}

The FOG stabilized system is designed to trace the given position and to isolate the stabilized platform from the carriers' motion. In actual FOG stabilized systems, frictions and clearances may make the platform work abnormally. In some systems, the platform's loads may not be constant or some other nonlinear factors may influence on the systems. In order to analyze the system easily, there will be some valid simplicities and approximated for the system model. So, the real FOG stabilized platform system is a nonlinear and time-variable object.

Like the classic PID controller, automatic disturbance rejection controller (ADRC) also could work without a precise model of a system. But ADRC have a better robustness by using nonlinear structures.

Based on background introduced above, one servo loop of a FOG stabilized platform will be taken as an example for research the application of ADRC.

\section{MODEL FOR THE SERVO LOOP}

Fig. 1 is the servo loop of a FOG stabilized platform. The servo loop is made up of two closed loops. The velocity loop is the inside one and the position loop is the outside one. In most position servo systems, an analog controller would be used for the velocity

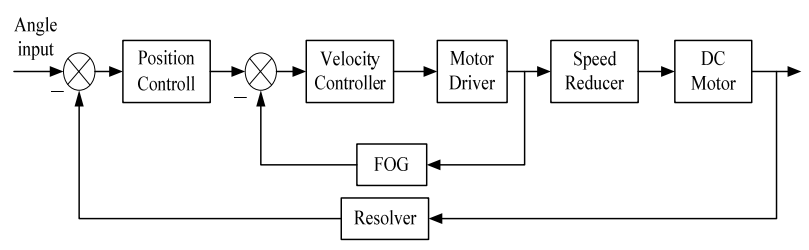

Figure 1. A servo loop of a FOG stabilized system

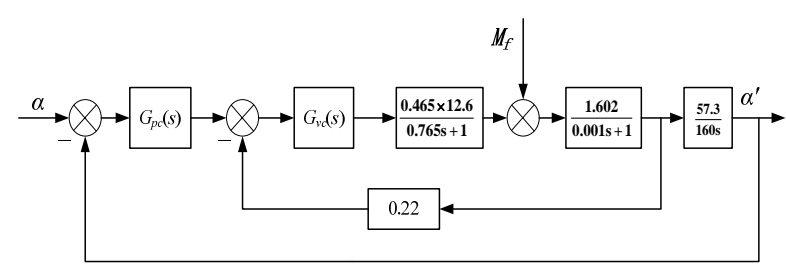

Figure 2. Model with actual parameters of the servo loop

loop to relieve the disturbances from the vary parameters or loads, and a digital controller would be used for the position loop to control the tracing characteristics. The model with actual parameters of a FOG stabilized platform is shown in Fig.2.

In order to focus on the control performances of the position loop, the compensated velocity loop would be often treat as a classic inertial unit whose transfer function was simplified to (1). Consequently, the open loop transfer function of the position loop was given by (2).

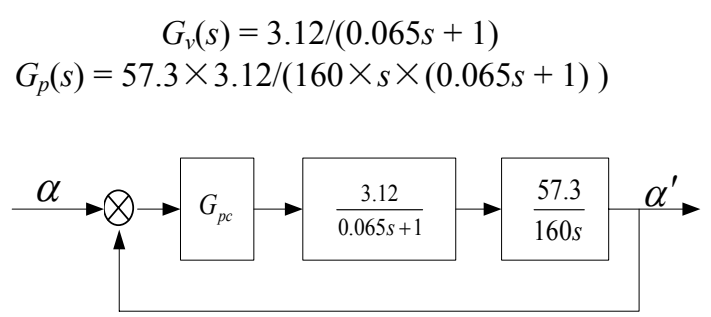

Figure 3. Position loop with one controller

Fig.3 was the closed position loop with one controller. The unit of angular signal was degree.

The indexes of control performances of this position loop should be designed as: setting time $\left(t_{s}\right) \leqslant 0.2 \mathrm{~s}$, and oscillations would be $1 \sim 2$ times, and overshoots would be $5 \% \sim 10 \%$. 


\section{Classic PID CONTROLleR}

According to the segment II, the PID controller of the position loop could be designed as (3).

$$
G_{p c}=\frac{k_{c}\left(T_{1} s+1\right)}{\left(T_{2} s+1\right)\left(T_{3} s+1\right)} .
$$

$k_{c}$ is the gain of the PID controller, and $T_{i}(i=1,2,3)$ is the time constant of the PID controller. According to simulation results, the best parameters were: $k_{c}=29$, $T_{1}=0.07 \mathrm{~s}, T_{2}=0.0157 \mathrm{~s}, T_{3}=0.00035 \mathrm{~s}$. Fig. 4 was the step response of the position loop. The system compensated by PID controller was stabilized. The dynamic performances of the system with PID controller could be satisfied to its designed goals.

If the open loop gain of PID controller was magnified by 10 times, the step response would be different. The new response curve was shown in Fig.5. Visibly, the amplitude of the oscillation of the output signal became larger and the times of the oscillation of output signal became more and the overshoot exceeded $32 \%$. FOG stabilized platform could not work normally in that condition.

A $50 \mathrm{~Hz}$ and 0.1 degree noise was introduced to this servo loop. As Fig.6 shown, though the setting time and overshoot had not been changed significantly, there were obvious vibrancies on the output steady signals.

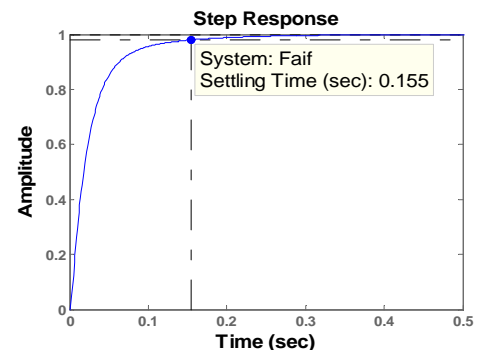

Figure 4. Step response of PID controller

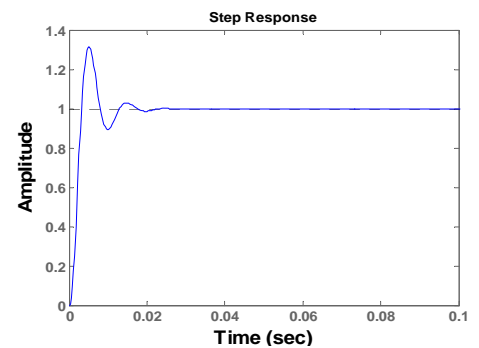

Figure 5. Step response of PID controller with changed parameters

\section{BASIC THEORY OF ADRC THEORY}

ADRC based on non-linear structure avoids some inherent defects of classical PID controller. ADRC has better adaptability and robustness. Generally, ADRC is made up of a tracking differentiator (TD) in the feed forward path,

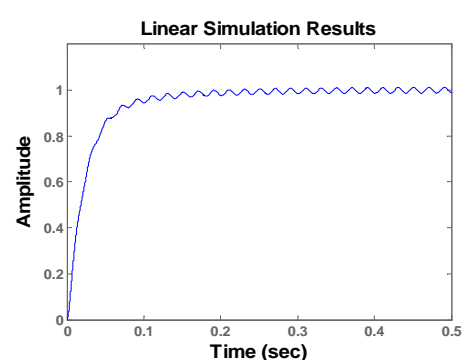

Figure 6. Step response of PID controller with noises added

an extended state observer (ESO), and a non-linear state error feedback (NSEF).

TD is used to preprocess the input angular signal. ESO is used to estimate state and perturbation of the system respectively. The error caused by uncertain system model is regarded as internal disturbances. The total disturbances consisting of internal disturbances and external disturbances work on the system as a whole. Comprehensive errors are measured directly. NSEF is used to obtain the compensation of disturbances. The efficiency of signal processing is improved by the nonlinearity combination of integral and differential of error signals. This type of control way has better robustness for system models and uncertainty units. Both the internal disturbances from models or parameters and the external disturbances can be compensated well. Therefore, this controller is named "Automatic Disturbance Rejection Controller".

In real systems, the performances of the stabilized platform are limited by the noisy given signal. Generally, the backward difference (BD) is often used to obtain the highquality differential signal from the noncontinuous noisy measured signal. In order to restrain the amount of this noise, the differential signal should be filtered. These conventional filters will introduce additional delays. If the filtered primary signal is used for synchronization purposes or time-critical feedback, those delays will be specially harmful.

The concrete principle of ADRC is depicted as: TD is used to process input reference and ESO is used to process system output. ADRC chooses an appropriate nonlinear combination of state errors according to a control rule. Fig.7 was the structure diagram of ADRC. A nth-order TD which offers all derivative tracking signal $\alpha_{1} \cdots \alpha_{n}$ of reference input $\alpha$ is a rapid and no overshoot transitional process. The $(\mathrm{n}+1)$ th-order ESO which is used for the compensating for internal and external disturbances and realizing the state feedback is made up of all the estimated state variables $z_{1} \cdots$ $z_{n}$ and all real-time disturbances $z_{n+1}$. The errors between the outputs of ESO and TD are actually the errors between the generalized output of the plant and the generalized input of the system. NSEF utilizes these errors to convert the uncertain nonlinear units to "integral cascade" units for control.

\section{A. Tracking differentiator}

Equation (4) is the discrete form: 


$$
\begin{aligned}
& \left\{\begin{array}{l}
v_{1}(k+1)=v_{1}(k)+h v_{2}(k) \\
v_{2}(k+1)=v_{2}(k)+h \operatorname{fst}\left(v_{1}(k)-v(k), v_{2}(k), r, h_{1}\right)
\end{array}\right. \\
& f_{s t}(\cdot)=-r s a t(g(k), \delta) \\
& \delta=h_{1} r, \delta_{1}=h_{1} \delta \\
& g(k)=\left\{\begin{array}{l}
v_{2}(k)-\operatorname{sgn}(s(k)) \times \frac{\delta-\sqrt{8 r\left|s(k)+\delta^{2}\right|}}{2},|s(k)|<\delta_{1} \\
v_{2}(k)+\frac{y}{h_{1}},|s(k)|<\delta_{1}
\end{array}\right. \\
& \operatorname{sat}(x, \delta)=\left\{\begin{array}{l}
\operatorname{sgn}(x),|x|<\delta \\
\frac{x}{\delta},|x|<\delta
\end{array}\right. \\
& e(k)=v_{1}(k)-v(k), s(k)=e(k)+h_{1} v_{2}(k)
\end{aligned}
$$

$v$ is an input vector, and $v_{1}$ is the tracking vector of $v$, and $v_{2}$ is the derivative vector of $v_{1}$, and $h$ is the integral step length, and $r$ is a parameter which determines the tracking speed.

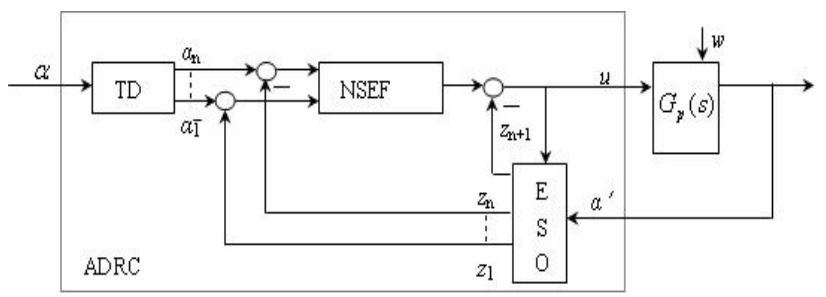

Figure 7. Block diagram of ADRC

\section{B. Expanded State Observer:}

In (5), the estimated state variables of the controlled object are $z_{1}(k)$ and $z_{2}(k)$. An expanded state $z_{3}(k)$ is used to calculate real-time sum of external disturbances and to estimate all uncertain internal disturbances.

$$
\begin{aligned}
& \left\{\begin{array}{l}
e(k)=z_{1}(k)-y(k) \\
z_{1}(k+1)=z_{1}(k)+h\left(z_{2}(k)-\beta_{01} e(k)\right) \\
z_{2}(k+1)=z_{2}(k)+h\left(z_{3}(k)-\beta_{02} \mathrm{fal}\left(e(k), \alpha_{2} / 2, \delta_{2}\right)+b_{0} u(k)\right) \\
z_{3}(k+1)=z_{2}(k)-h \beta_{03} \mathrm{fal}\left(e(k), \alpha_{2} / 4, \delta_{2}\right)
\end{array}\right. \\
& \left\{\begin{array}{l}
e_{1}(k)=v_{1}(k)-z_{1}(k) \\
e_{2}(k)=v_{2}(k)-z_{2}(k) \\
u_{0}(k)=\beta_{1} \mathrm{fal}\left(e_{1}(k), \alpha_{3}, \delta_{3}\right)+\beta_{2} \mathrm{fal}\left(e_{2}(k), \alpha_{3}, \delta_{3}\right) \\
u(k)=u_{0}(k)-z_{3}(k) / b_{0}
\end{array}\right.
\end{aligned}
$$

The nonlinear operator fal $(\bullet)$ is given by

$$
\operatorname{fal}(\varepsilon, \alpha, \delta)=\left\{\begin{array}{l}
|\varepsilon|^{\alpha} \operatorname{sgn}(\varepsilon), \quad|\varepsilon|>\delta \\
\varepsilon / \delta^{1-\alpha}, \quad|\varepsilon| \leq \delta
\end{array}\right.
$$

Because the extended state can track the actual actions of the whole disturbances, the nonlinear ESO could compensate disturbances well and have high robustness to the variations of the model parameters.

\section{Non-linear State Error Feedback}

Utilizing the error between the output of the TD and the output of the ESO, NSEF could combine non-linear control and unknown disturbances compensation together.

In (6) and (7), $u$ is a control vector, and $y$ is the output vector, $\beta_{01}, \beta_{02}, \beta_{03}, \beta_{1}$ and $\beta_{2}$ are all adjustable parameters, and $b_{0}$ is the coefficient of the control vector of state space matrix.

The expression $f a l(\varepsilon, \alpha, \delta)$ is used to achieve the automatic gain adjustment. It is proved in reference [5] that the variation of the gains results in rapid transit with elaborate damping. When the errors become bigger, the proportional gain can automatically generate a bigger compensation to adjust the output of the controlled system to trace rapidly. When the errors disappear, the derivative gain and the proportional gain can also automatically adjust the output of the controlled system to prevent big overshoots and excessive oscillations.

\section{Application Of AdRC In Fog Stabilized System.}

The state space expression of the position loop is:

$$
\left\{\begin{array}{l}
\dot{x}_{1}=x_{2} \\
\dot{x}_{2}=-15.38 x_{2}+17.13 u \\
y=x_{1}
\end{array}\right.
$$

Consequently, an ADRC is designed based on expression (8). Fig.8 was a two-order ADRC including Two-order TD, three-order ESO and NSEF. $\alpha$ is the error vector, $G_{p}(s)$ is the stabilized system, $\omega_{f}$ is a disturbance vector, and $\alpha^{\prime}$ is the output vector.

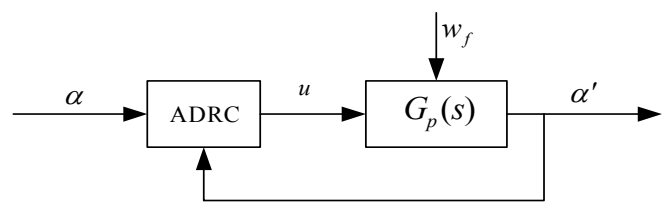

Figure 8. ADRC used in position loop

According to result of simulations, the control parameters of ESO, TD and NSEF are fixed: $r=6006, h_{1}=0.56, \alpha_{2}=$ $0.04, \delta_{2}=0.04, \beta_{01}=0.15, \beta_{02}=0.62, \beta_{03}=0.16, \beta_{1}=0.046$, $\beta_{2}=0.002, \alpha_{3}=0.016, \delta_{3}=0.11$.

The step response curve of this stabilized system without the effects of model perturbation and external disturbances was shown in Fig. 9. The output curve had little overshoot, and the setting time (the error limit $\Delta=2 \%$ ) is only $126 \mathrm{~ms}$. The same conditions with PID controller were also simulated. All parameters of model were not changed except the gain of open loop transfer function was magnified by 10 times. Fig.10 is the step response curve of the output. Comparing 
Fig.9 and Fig.10, it was significant that the performance of ADRC would not be prominently affected on the condition that the parameters of control model were changed. The excellent robustness of ADRC is shown.

At last, a step input and a high-frequency sine disturbance were introduced to the position loop together. The response curve of output is shown in Fig. 11. The changes between the responses of output with disturbances and that with no disturbances were not significant. But the fluctuations were slight.

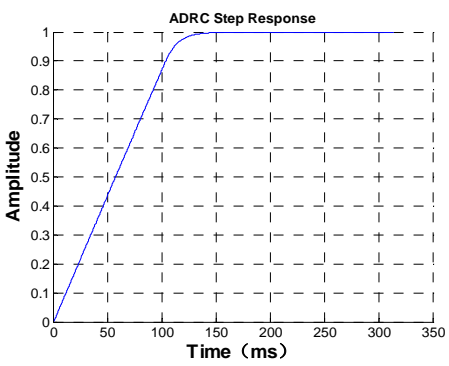

Figure 9. Step response of ADRC

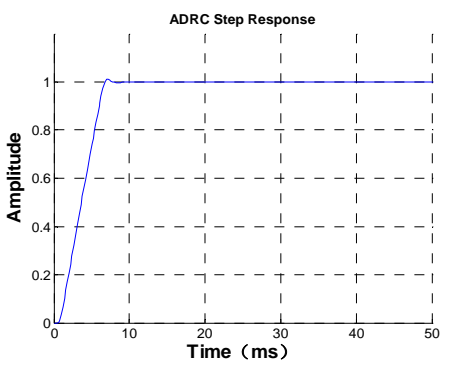

Figure 10. Step response of ADRC with the parameters changed

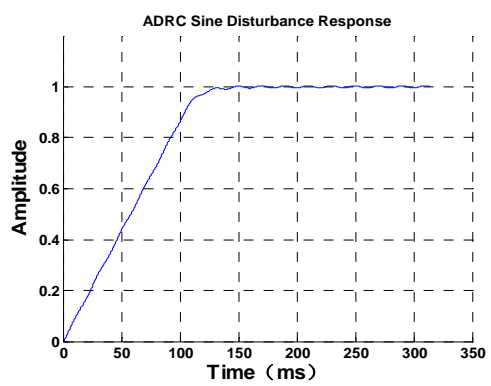

Figure 11. Step response of ADRC with the sinusoidal disturbances

\section{CONCLUSION}

As the above simulation results shown, the responses with ADRC had no oscillation, no significant overshoot, faster responses, and higher steady state precision. ADRC is not sensitive to the changes of the model parameters, and has superior ability to restrain the sinusoidal disturbances. Compared to simulations results of PID controller, the performances of ADRC are more excellent than classical PID obviously. ADRC is very suitable for highly precise FOG stabilized systems.

\section{ACKNOWLEDGMENT}

The authors are very grateful to the anonymous referees for the numerous remarks and suggestions which have lead to significant improvements of this paper. This paper is supported by the Fundamental Research Funds for the Central Universities (HEUCF041329).

\section{REFERENCES}

[1] Z. Q. Han, "Auto-disturbances-rejection Controller and Its Applications," Control and Decision, no.1, pp.19-23, 1998.

[2] Z. Q. Han, W. Wang, "Nonlinear tracker-differentiator," Journal of Systems Science and Mathmatical sciences, no.2, pp.177-183, 1994.

[3] Z. Q. Han, "a Class of Expanded State Observer of Uncertain object ," Control and Decision, no.1, pp.85-88, 1995.

[4] Z. Q. Han, "nonlinear state error feedback-NLSEF," Control and Decision, no.5, pp.221-225, p.231, 1995.

[5] Y. X. Su, C. H. Zheng, B. Y. Duan, "Automatic disturbances rejection controller for precise motion control of permanent-magnet synchronous motors," Industrial Electronics, vol.52, pp. 814 - 822, Jun. 2005.

[6] H. K. Le, Z. F. Tan, et al, "Ship Swinging and Control and Stabilizing of the Sonar Array Attitude," Journal of Harbin Engineering University, no.5, pp.36-42, 1997.

[7] D. Zhao, Z. F. Tan, et al, "Compound Control of Position Stabilization System for Sonar Array," Marine Electric \& Electronic Technology, no.2, pp.23-27, 2003.

[8] G. Feng, L.P. Huang, D.Q. Zhu, "Nonlinear auto-disturbance rejection controller for induction motor," IECON Proceedings (Industrial Electronics Conference), no.3, pp.1509-1514, 1998.

[9] X. Z. Liu, J. Wu, "Design of permanent-magnet linear synchronous motor control system based on auto-disturbance-rejection controller," Transactions of China Electrotechnical Society, vol.19: pp.6-11, 2004.

[10] Z. Q. Gao, S. H. Hu, F. G. Jiang, "A novel motion control design approach based on active disturbance rejection Source," Proceedings of the IEEE Conference on Decision and Control, no.5, pp4877-4882, 2001. 